\section{Frühe Haselpollen}

Schon in den ersten Dezembertagen 2006 ist es an mehreren Orten in Deutschland zum Auftreten von Haselnusspollen gekommen. Dies ist ungewöhnlich früh, der Flug von Haselpollen beginnt regelhaft erst im Januar. Die Stiftung Deutscher Polleninformationsdienst (PID) wertet diesen frühesten bisher dokumentierten Beginn des Haselnusspollenflugs als Ausdruck der warmen Wetterlage und möglicherweise des diskutierten Klimawandels.

\section{Abfahrt für Asthmatiker}

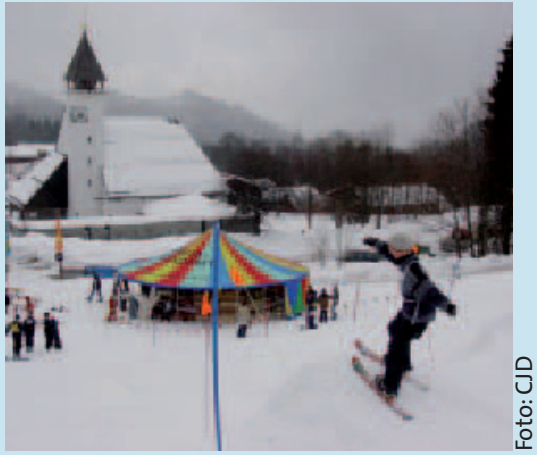

Das CJD Asthmazentrum Berchtesgaden richtet am 28. Februar 2007 einen zehnstündigen Skimarathon für asthmakranke Kinder und Jugendliche aus. „Im Winter ist die trockene, kalte Luft zwar ein Belastungsfaktor", so Dr. Gerd Schauerte vom CJD Asthmazentrum, „dennoch können Asthmatiker Ski fahren und im Schnee Spaß haben, wenn sie die Verhaltensregeln beachten, die jeder Asthmatiker im Asthma-Verhaltenstraining lernt". Teilnehmen können alle Asthmatiker, die über ausreichendes skifahrerisches Können verfügen.

Ärzte, die ihren Patienten auf die Veranstaltung hinweisen wollen, erhalten online unter www.cjd-asthma.de oder telefonisch unter (o 86 52) 60 oo-1 61 weitere Informationen.

\section{Exzellenz in München}

Die Klinik und Poliklinik für Dermatologie und Allergologie am Biederstein, Klinikum rechts der Isar, Technische Universität München, wurde in einem externen Audit im Rahmen des GA2LEN-Programms (Global Allergy and Asthma European Network) der Europäischen Union einer Qualitätskontrolle unterzogen. Dem Ergebnis zufolge erfüllt die Klinik die Kriterien des europäischen ExzellenzNetzes für "Clinical Care in Allergy“.

\title{
Kinderpneumologen tagen in München
}

$\mathrm{V}$ om 22. bis zum 24. März 2007 veranstaltet die Gesellschaft für Pädiatrische Pneumologie (GPP) in München ihre 29. Jahrestagung. Dem Kongress vorgeschaltet sind am 21. März Postgraduiertenkurse mit begrenzter Teilnehmerzahl. Durch ihr breit gefächertes Programm bietet die Tagung Möglichkeiten zur praktischen und theoretischen Weiterbildung auf allen Gebieten der pädiatrischen Pneumologie. Ein besonderes Anliegen ist es, die neuesten Kenntnisse aus den Randbereichen der pädiatrischen Pneumologie, wie etwa der Radiologie, der pädiatrischen Intensivmedizin, der Molekularbiologie und der Immunologie, darzustellen.

Vor allem an niedergelassene Ärzte wendet sich das praxisorientierte Fortbildungsangebot am Mittwoch und am Samstag. Es ist aber auch zugeschnitten auf die Ansprüche der Kollegen, die sich in der Weiterbildung zum pädiatrischen Pneumologen befinden, und für alle anderen Berufsgruppen, die Kinder und Jugendliche mit Atemwegs- und Lungenerkrankungen betreuen.
29. GPP-Tagung auf einen Blick

Kongresstermin

Postgraduiertenkurse: 21./22. März 2007

Hauptprogramm: 22.-24. März 2007

\section{Wissenschaftliche Leitung}

Prof. Dr. Matthias Griese

Dr. von Haunersches Kinderspital der Universität München

Kongressorganisation

Wurms \& Partner PR GmbH, Bernrieder Straße 4, 82327 Tutzing

Tel.: (o 8158) 99 67-0

Fax: (0 81 58) $9967-29$

E-Mail: info@wurms-pr.de

\section{Kongress-Website}

www.gpp-congress-2007.de

Als besonderen Service bieten die Veranstalter in Zusammenarbeit mit der Deutschen Bahn AG ein günstiges Kongressticket an. Einzelheiten dazu sowie auch das komplette Kongressprogramm finden sich unter www.gpp-congress2007.de auf der Kongress-Website. iw

\section{Auf der Suche nach dem Superstaub}

E. ine Kindheit auf dem Bauernhof schützt vor Allergien und Asthma, das belegen inzwischen mehrere epidemiologische Untersuchungen. Was aber genau die protektiven Faktoren in der Landluft sind, ist noch weitgehend unklar. Licht ins Dunkel soll jetzt eine große Fragebogenaktion im Rahmen des europäischen GABRIEL-Projektes bringen. Dazu hat ein Team um Prof. Dr. Erika von Mutius von der Dr. von Haunerschen Kinderklinik in München im vergangenen Dezember Fragebögen an rund 40.000 bayerische Grundschüler ausgegeben, in denen allgemeine $\mathrm{Ge}$ sundheitsdaten erfragt wurden und um Teilnahme an der Hauptstudie gebeten wurde. Dort sollen dann bei den beteiligten Kindern Blutproben genommen sowie Staubproben im Wohnbereich bzw. auch im Stall gesammelt werden. Bei der
Auswertung der Daten ergibt sich dann, so die Hoffnung der Forscher, eine Korrelation zwischen festgestellten Sensibilisierungen und der spezifischen Staubzusammensetzung. Weitere Informationen gibt es unter http://gabriel.web.med.unimuenchen.de im Internet. red

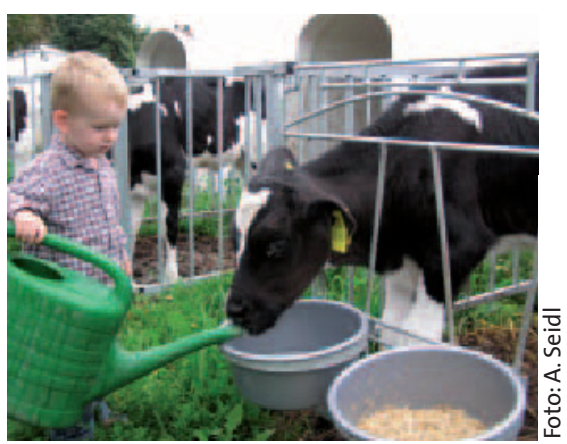

Ein Tauschgeschäft: Wasser gegen Allergieprotektion? 